PIERRE DUYSINX AND MICHAEL BRUYNEEL

\title{
RECENT PROGRESS IN PRELIMINARY DESIGN OF MECHANICAL COMPONENTS WITH TOPOLOGY OPTIMIZATION
}

\section{INTRODUCTION}

Since 10 years topology optimization has been trying to bring an efficient answer to the automatic choice of the morphology of mechanical components, i.e. the number and the relative positions of the holes in the structural domains, the number and the nature of the structural members, their connectivity and the character of the connecting joints. This problem is one of the main questions to be addressed during the preliminary design phase of mechanical and structural components. Up to now, the selection of the mechanical morphology has been let to engineers' experience or to their intuition (which is even worse sometimes). With topology optimization the choice of the morphology can now rely on rational arguments and can be driven with the help of mathematical tools. This has two advantages. At first topology optimization can facilitate the automation of the preliminary design, but it can also improve substantially the performance of new mechanical products, that is, topology optimization can propose original and innovative solutions to engineering problems.

For a long time topology optimization has been based on 'compliance type' arguments as in the pioneer paper of Bendsøe and Kikuchi (1988). This kind of formulation has produced quite interesting results in many problems especially because controlling the displacements under the loads is generally good for deflection control and because, for one load case, compliance minimization leads to a fully stressed design nearly everywhere in the structure (Rozvany and Birker, 1994, Rozvany, 1996 and 1998). However recently new results showed that topology predictions can not always be reduced to a compliance minimization. For example Rozvany and Birker (1994) have demonstrated that, for trusses, topology optimization can lead to different results when there are several load cases, different stress limits in tension and compression, or when there are several materials involved.

This key role of stress constraints has to be demonstrated in the framework of topology optimization of continuum structures, for several load cases and when unequal stress limits in tension and compression are considered.

The present developments continue along the work that was done in Duysinx and Bendsøe (1998) to introduce stress constraints in topology optimization of continuum structures. At first an integrated (i.e. global) relaxed stress constraint is 
introduced. A second contribution is the generalization of the von Mises equivalent stress to other quadratic criteria that are able to cope with unequal stress limits.

In addition to these mechanical constraints, one can also introduce geometric constraints on the material distribution. Besides the very classic area/volume constraint, the perimeter constraint offers an elegant way to control the complexity of the geometry that comes out from the optimization procedure. A first contribution has been made to cope with this difficult constraint in the mathematical programming solution procedure. A second development aimed at extending the existing perimeter measure to 3-D and axisymmetric problems.

\section{FORMULATION OF TOPOLOGY PROBLEM}

Shape optimization of structures without any a priori on the structural topology can be achieved by formulating the problem as an optimal material distribution on a given design domain (see Bendsøe, 1995). In order to solve numerically the optimal material distribution problem, the design domain is divided into finite elements and a density variable is attached to each element. The optimal material distribution problem could be solved as a discrete valued problem, but this approach is very complicated because of its highly combinatorial nature. Here a simplified formulation is considered. We allow the density parameter to run continuously from void to solid via all intermediate densities. The modeling of effective intermediate densities properties is based on a power-law model (also called SIMP model as in Rozvany et al., 1992). This model is extremely popular for solving industrial applications because of its simplicity. If the script * denotes effective properties of the porous material and the index 0 is relative to the solid material properties, the effective Young's modulus $E^{*}$ is given in term of the density $\rho$ by $E^{*}=\rho^{p} E^{0}$. The exponent $p>1$ is introduced to penalize the intermediate densities in order to end up with 'black and white' designs.

This continuous formulation presents the advantage to allow the use of sensitivity analysis and mathematical programming algorithms to solve the problem in an efficient way (see for example Duysinx, 1997). However because the power law model is an approximation of composite materials the design problem is illposed, i.e. the numerical solutions are mesh-dependent. To overcome the difficulty, we use here a restriction method of the design space based on a bound over the perimeter (Haber et al., 1996).

In addition to stiffness properties, if one wants to consider stress constraints in continuous topology optimization, one also needs the definition of a relevant stress measure in the porous composites. Modeling of strength properties can be made by following the approach developed in Duysinx and Bendsøe (1998). An overall stress measure controls the stress state in the microstructure. In the framework of the SIMP model, Duysinx and Bendsøe (1998) showed that a power-law model with the same power $p$ is a consistent model for the micro-stresses $\sigma_{i j}$. Therefore if the failure is predicted by a quadratic overall failure criterion like von Mises equivalent criterion, 
the first failure in the microstructure of the porous composites is predicted by the following criterion in terms of effective stresses:

$$
\sigma_{i j}=\sigma_{i j}^{*} / \rho^{p} \quad \Rightarrow \quad\left\|\sigma^{e q}(\rho)\right\|={ }^{*} \sigma^{e q} / \rho^{p} \leq T
$$

\section{PERIMETER CONSTRAINT}

The perimeter constraint was originally introduced by Haber et al. (1996) to assure the regularization of topology problems (even with $0 / 1$ variables and with intermediate density penalization). In addition to that property it was shown that the perimeter method is a very elegant solution to control the complexity of topology solutions, and thereby, to introduce an indirect control over the manufacturability of the solution (Figure 1). When working with distributions of porous materials, the intuitive geometric measure of perimeter can be replaced by the total variation of the density, which is just the density jumps across the finite element edges when density discretization is constant on each element.
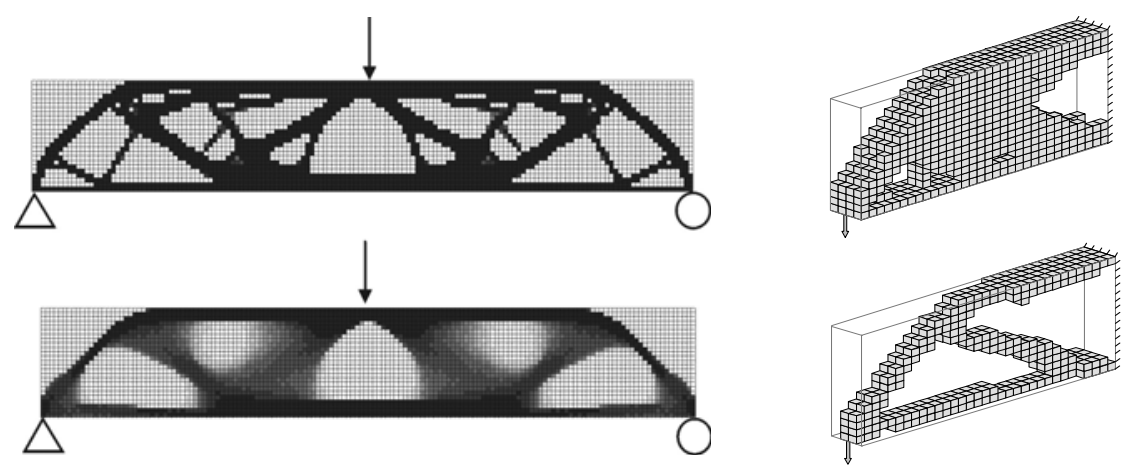

Figure 1. Using perimeter constraint for the control of the complexity of topology solutions.

Perimeter constraints showed to be very difficult to control within the mathematical programming solution procedure. A first contribution was realized to cope with this type of constraint (Duysinx, 1997). Another part of the work has been devoted to extend the existing measure, which was restricted to planar geometries, to 3-D (Bruyneel, 1998) and axisymmetric structures (Figure 1b).

\section{STRESS CONSTRAINTS}

At first it is interesting to remind the reader with a very important difficulty that arises when dealing with stress constraints in topology optimization. As it has been shown in Kirsch (1990) and in Cheng and Jiang (1992), topology optimization with 
stress constraints is subject to the 'singularity phenomenon'. At short the paradox comes from the fact that the optimization procedure is often unable to remove or to add some vanishing members without violating the stress constraints although one would end up with a perfectly feasible design if they were removed or added. From a mathematical point of view, the classical algorithms are unable to reach some optimum configurations because of the degeneracy of the design space. In order to turn around the difficulty, one has to use a perturbation technique of the stress constraints, generally known as the $\varepsilon$-relaxation technique (Cheng and Guo, 1997) that results in a relaxation of the stress limits in the low-density regions. In this paper we use the following $\varepsilon$-relaxed formulation of the overall stress criterion:

$$
\frac{1}{T} \frac{\sigma^{e q}}{\rho^{p}}-\frac{\varepsilon}{\rho}+\varepsilon \leq 1
$$

This relaxed formulation of the stress constraints is considered in the numerical solution of the topology optimization problem with stress constraints. The solution procedure requires the solution of a sequence of perturbed problems with decreasing values of the parameter $\varepsilon$.

\section{INTEGRATED STRESS CONSTRAINTS}

In Duysinx and Bendsøe (1998) the stress is treated as a local constraint, i.e. for every finite element. This formulation offers a full control of the stress state, but it also dramatically increases the size of the optimization problem and thus the computation time of the solution. Here we propose an alternative approach based on an integrated version of the $\varepsilon$-relaxed failure criterion. The global stress constraint (i.e. integrated constraint) simplifies the computational complexity of the optimization problem at the expense of a weaker control of the local stress state. A key issue is to take into account the 'singularity phenomenon' of the stress constraints. So an original aspect is to include simultaneously the $\varepsilon$-relaxation technique that alleviates the singularity phenomenon and the use of effective stress criteria into the global stress constraint.

We propose to consider two global measures of the relaxed distributed stress criterion. The first global constraint is the ' $q$-norm' of the relaxed stress criterion (3); the second one is the 'q-mean' of the relaxed stress criterion (4).

$$
\begin{aligned}
& {\left[\sum_{e=1}^{N}\left(\max \left\{0, \frac{1}{T} \frac{\left({ }^{*} \sigma^{e q}\right)_{e}}{\rho_{e}^{p}}-\frac{\varepsilon}{\rho_{e}}+\varepsilon\right\}\right)^{q}\right]^{1 / q} \leq 1} \\
& {\left[\frac{1}{N} \sum_{e=1}^{N}\left(\max \left\{0, \frac{1}{T} \frac{\left({ }^{*} \sigma^{e q}\right)_{e}}{\rho_{e}^{p}}-\frac{\varepsilon}{\rho_{e}}+\varepsilon\right\}\right)^{q}\right]^{1 / q} \leq 1}
\end{aligned}
$$


To prevent negative valued relaxed criteria from contributing to the norms, one must consider the maximum value of the relaxed stress criterion and zero. Negative values of the relaxed criterion appear only for low stressed elements and thus can be truncated without influencing the global constraint. Furthermore, the function remains continuous up to derivative ' $q-1$ ', so that the constraint remains sufficiently smooth. A major advantage of ' $q$-norm' and ' $q$-mean' functions comes from the fact that they make possible to bound the maximum value of the relaxed criterion by upper or by lower values. The ' $q$-norm' is an upper bound of the maximum value of the criterion while the ' $q$-mean' is a lower bound of the maximum local value of the criterion. In addition this gap closes to 0 when ' $q$ ' value is going to infinity. So sharp bounding needs to take high values of ' $q$ ' parameter. However in practice, we take $q=4$ because it allows to control weakly the maximum value of the stress criterion while avoiding ill-conditioned optimization problems.

\section{TREATMENT OF UNEQUAL STRESS CONSTRAINTS}

Up to now topology optimization with stress constraints was based on the quadratic von Mises criterion. This criterion is very usual, because it predicts very precisely failure for ductile materials and metals, which are commonly used in mechanical engineering. However von Mises criterion is unable to predict real-life designs when the structure is made of materials with unequal stress limits like concrete or composite materials. One can also remember that thin structural members, like cables or thin sheets, are not able to sustain high compressive loads because of buckling. An indirect procedure to take into account this buckling constraint in the preliminary design phase consists in restricting the compressive loads by reducing the stress limit in compression. From a practical point of view these different behaviors in tension and compression result in quite specific designs. In order to predict layouts that take into account different behaviors in tension and compression, one requires particular failure criteria that are able to cope with unequal stress limits.

To this end, two quadratic criteria have been selected and implemented. They both introduce a dependency on the first invariant of the stresses. The first equivalent failure criterion is the Raghava criterion (5a), which is generally used with adhesive materials. An interesting alternative to Raghava is the Ishai criterion (5b). These stress criteria and the related equivalent stress can be written as:

$$
\sigma_{R A G}^{e q}=\frac{J_{1}(s-1)+\sqrt{J_{1}^{2}(s-1)^{2}+12 J_{2 D} s}}{2 s} \leq T \quad \sigma_{I S H}^{e q}=\frac{(s+1) \sqrt{3 J_{2 D}}+(s-1) J_{1}}{2 s} \leq T
$$

$T$ and $C$ are the stress limits (in absolute value) respectively in tension and compression, and ' $s$ ' is the ratio between the stress limits in compression and in tension: $s=C / T$. The criteria are written in terms of the first stress invariant $J_{1}=\sigma_{i i}$ and the second deviator stress invariant $J_{2 D}=0.5 s_{i j} s_{i j}$. The presence of the second invariant is obvious because the criteria have to render the von Mises criterion when the stress limits are equal. The first invariant is related to the hydrostatic pressure 
and its presence in the criteria is essential to introduce the dependence upon the sign of the stress state and so the different behaviors in tension and compression.

\section{THE 3-BAR TRUSS EXAMPLE}

Despite its extreme simplicity, the 3-bar truss problem is very well suited to illustrate several concepts related to topology optimization with stress constraints. The geometry of the problem is given in Figure 2. The sizes and material data of the benchmark are normalized: $\mathrm{L}=1 \mathrm{~m}, \mathrm{~W}=2.5 \mathrm{~m}, \mathrm{E}=100 \mathrm{~N} / \mathrm{m}^{2}, \mathrm{v}=0.3$. Three load cases (with different magnitudes and orientations) are applied at the center of the free edge. The design domain is meshed with 50 x 20 finite elements. This means that for local stress constraints one has to deal with $3 \times 1000$ restrictions. The volume bound is set to $25 \%$ of the design domain area.

At first the minimum compliance design is studied (Figure 3a). The optimal topology is a 2-bar truss. Compliances for the 3 load cases are $73.3 \mathrm{Nm}$, but stress level is quite high. Using von Mises criterion, maximum value of the local criterion varies from $228 \mathrm{~N} / \mathrm{m}^{2}$ to $571 \mathrm{~N} / \mathrm{m}^{2}$ per load case. In a second optimization run one consider local von Mises stress constraints with a stress limit of $150 \mathrm{~N} / \mathrm{m}^{2}$ and one gets the topology of Figure $3 \mathrm{~b}$ which is a 3-bar truss. The compliances of the minimum stress design solution are a bit bigger than minimum compliance solution (91.2 Nm, 45.6 Nm and 45.0 Nm for load cases 1, 2 and 3).

The conclusion that can be drawn from the comparison of these two optimization runs is that compliance design and minimum stress design can lead to different topology solutions when there are several load cases.

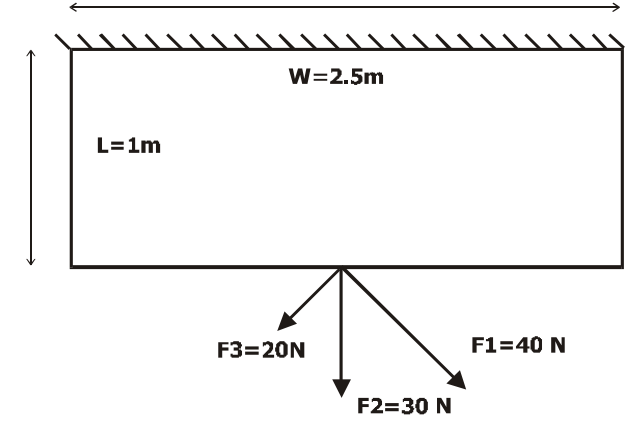

Figure 2. Geometry of the '3-bar truss' problem.

One can further compare the local stress constraint formulation and the alternative approaches based on global restrictions ('q-mean' and 'q-norm') with optimization runs presented in Figures 4. One has the solution when minimizing the volume of material with a (heuristic) bound of $92 \mathrm{~N} / \mathrm{m}^{2}$ over the ' $q$-mean' of von Mises stresses (Figure 4a). The optimal area is $0.56 \mathrm{~m}^{2}$. In Figure $4 \mathrm{~b}$, the bound over ' $q$-norm' is set to $500 \mathrm{~N} / \mathrm{m}^{2}$ and the optimal area is $0.62 \mathrm{~m}^{2}$. Topology results, which are 2-bar truss designs, look more like the compliance design, because we lose the 
control over the local stress state. However maximum local stress values are much lower than in compliance design. For ' $q$-mean' one has the following maximum von Mises stresses: $237 \mathrm{~N} / \mathrm{m}^{2}, 215 \mathrm{~N} / \mathrm{m}^{2}$, and $207 \mathrm{~N} / \mathrm{m}^{2}$ for the first, second and third load cases. For the 'q-norm' on has a maximum von Mises stresses of 230 N/m², 235 $\mathrm{N} / \mathrm{m}^{2}$, and $231 \mathrm{~N} / \mathrm{m}^{2}$ respectively for the three load cases. The weaker control over stress level allows to save one or two orders of magnitude in the optimizer solution time compared to the local stress approach. Finally one can appreciate the effect of unequal stress limits in tension and compression and Ishai criterion with results presented in Figures 5. The two results exhibit globally a 2-bar truss topology as for minimum compliance design, even though the geometry is quite different. When material is very resistant to compressive stresses, the middle and the left bars merge. In the opposite way when material is very good in tension, the optimal structure looks like a cable structure. This shows that real characteristics of the problem, here the unequal properties in tension and compression, may strongly influence topology results.
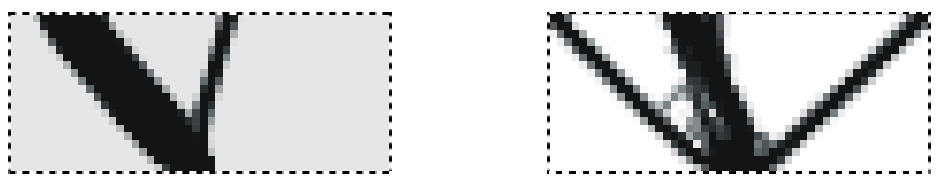

Figure 3. a) Min. compliance solution and b) Min. of the max. of the local von Mises stress.
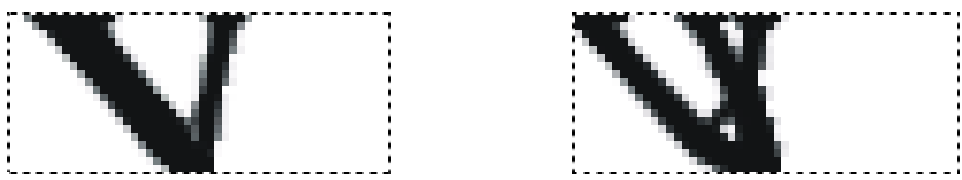

Figure 4. a) Min. of ' $q$-mean' of stress criteria and b) Min. of ' $q$-norm' of stress criteria.
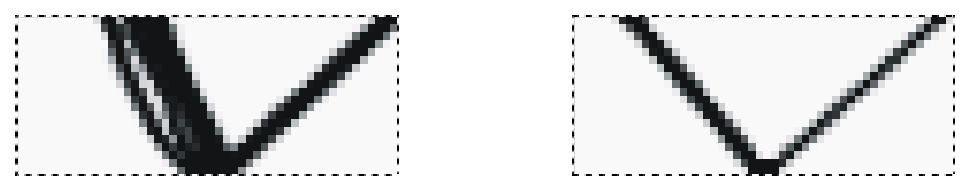

Figure 5. Minimum of maximum of local Ishai criteria a) $T=150 \mathrm{~N} / \mathrm{m}^{2}, C=450 \mathrm{~N} / \mathrm{m}^{2}$ and b) $T=450 \mathrm{~N} / \mathrm{m}^{2}, C=150 \mathrm{~N} / \mathrm{m}^{2}$.

\section{CONCLUSIONS}

Our work has extended the number of mechanical criteria, which can be considered in topology optimization design. For static analysis, we can consider compliance and deformation energy criteria, displacements constraints and stress constraints. Of course these constraints can be considered for one or several load cases. Because of their local character, the treatment of stress constraints in every finite element can be 
very cumbersome. So local stress constraints can be replaced by integrated (global) constraints with a certain success. Different stress criteria can also be taken into account. The classic von Mises criterion can be substituted by Raghava or Isaï criteria for unequal stress limits in tension and compression.

In addition to mechanical constraints, one can also introduce geometric constraints on the material distribution: area/volume and perimeter constraint. Perimeter constraints are very interesting because they offer an elegant way to control the complexity of the geometry that comes out from the optimization procedure. Several works were realized to extend the 2-D perimeter measure for plane structure to 3-D and axisymmetric problems.

As illustrated in the numerical application of this paper, the optimal structural morphology can be sensitive to the design criteria that are considered in the optimization. More generally the experience gained while solving optimization problems with the different design criteria presented herein leads to reconsider the general idea that topology optimization, as a preliminary design tool, can be based on a restricted number of design criteria, especially compliance. On the contrary we believe that a larger set of criteria like stress constraints have to be introduced in topology design in order to predict really optimal morphologies of structural components.

\section{AFFILIATIONS}

University of Liège, Mechanical Institute B52, B-4000, Liège, Belgium.

\section{REFERENCES}

Bendsøe M.P. \& Kikuchi N. (1988). Generating optimal topologies in structural design using a homogenization method. Comp. Meth. in Appl. Mech. and Eng.,71, 197-224.

Bendsøe M.P. (1995). Optimization of structural topology, shape, and material. Berlin: Springer Verlag.

Bruyneel M. (1998). Le périmètre en optimisation topologique de structures tridimensionnelles. University of Liège, Aerospace Laboratory. Report OF50.

Cheng G.D. \& Jiang Z. (1992). Difficulties in truss topology optimization with stress constraints. Eng. Optim., 20, 129-148.

Cheng G.D. \& X. Guo. (1997). \&-relaxed approach in structural topology optimization. Struct. Opt., 13, 258-266.

Duysinx P. (1997). Layout optimization: a mathematical programming approach. Danish Center for Applied Mathematics and Mechanics. DCAMM Report No 540.

Duysinx P. \& M.P. Bendsøe. (1998). Topology optimization of continuum structures with local stress constraints. Int. J. Num. Meth. Engng., 43, 1453-1478.

Haber R.B, Jog C.S. \& M.P. Bendsøe. (1996). A new approach to variable-topology shape design using a constraint on perimeter. Struct. Opt., 11, 1-12.

Kirsch U. (1990). On singular topologies in optimum structural design. Struct. Opt., 2, 133-142.

Rozvany G.I.N,. Zhou M. \& Birker T. (1992). Generalized shape optimization without homogenization. Struct. Opt., 4, 250-252.

Rozvany G.IN. \& Birker T. (1994). On singular topologies in exact layout optimization. Struct. Opt., 8, 228-235.

Rozvany G.I.N. (1996). Some shortcomings in Michell’s truss theory. Struct. Opt., 12, 244-250.

Rozvany G.I.N. (1998). Exact analytical solutions for some popular benchmark problems in topology optimization. Struct. Opt., 15, 42-48. 